\title{
LA-7855-MS
}

Informal Report

\section{Neutron Scattering Cross Sections for ${ }^{242} \mathrm{Pu}$}

$\frac{\frac{\pi}{E}}{\frac{0}{0}}$

$\overleftarrow{0}$

$\frac{2}{\omega}$

党

론

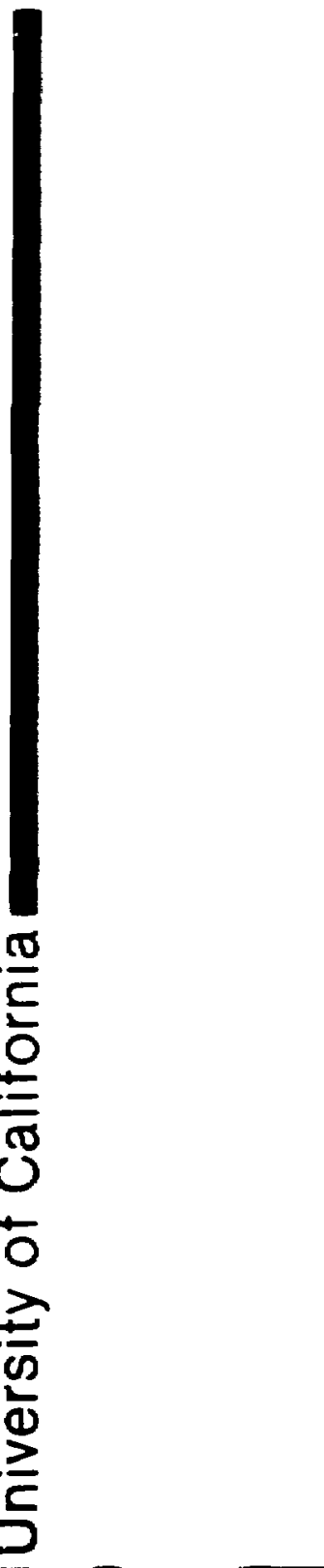


L.A.7B55.MS

Intormal Report

UC. 34c

Issued: October 1979

\title{
Neutron Scattering Cross Sections for ${ }^{242} \mathrm{Pu}$
}

\author{
D. M. Drake \\ M. Drosg* \\ P. Lisowski \\ L. Veeser
}

\section{MASTER}

-Irstanes thr Experimental Physics, Strudihotg. 4. A-1090, Wien, Austria.
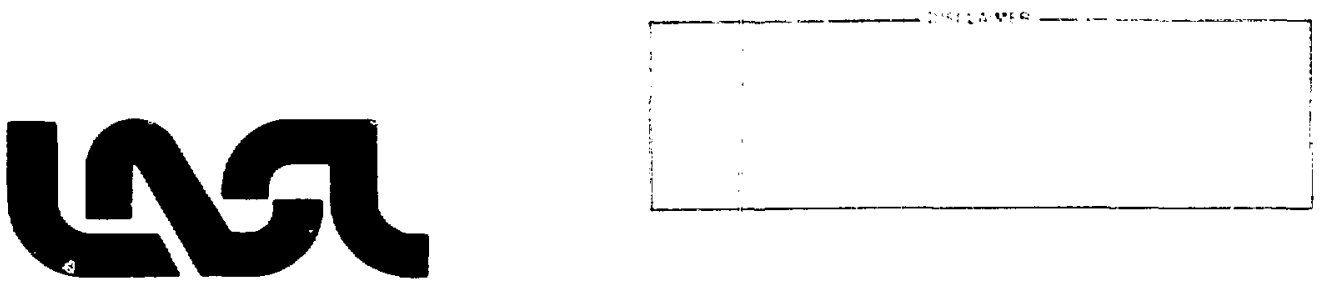


\title{
VELTROAi SCATTERI:ig CROSS SECTIONIS FOR ${ }^{242} \mathrm{Pu}$
}

by

¿. A. Jrake, $\because$. Orosg, P. Lisowski, and L. Veeser

\begin{abstract}
Lifferentid l cross sections for neutron scattering fro. "Pu are reported for 10 angles at each of three incident neutron energies: $0.57,1.0$, and $1.5 \mathrm{MeV}$.
\end{abstract}

\section{IHTRODUCTIO:}

We measured nelitron scattering cross sections of ${ }^{242} \mathrm{pu}_{\mathrm{u}}$ for incident neutrons of $0.57,1.0$, and $1.5 \mathrm{MeV}$ and 10 angles. These cross sections can be used to determine optical rodel parameters that subsequently can be employed to calculate neutron scattering from 242 pu over a large energy range.

\section{EXPERIMENTAL ARRANGEMENT}

Tine-of-flight (TOF) techniques that incorporate a pulsed neutron beam and a fast liquid scintillator were used to measure the emitted neutron energy spectra. Figure 1 shows the experimental arrangement.

A. Ne tron Sources

Two different neutron-producing reactions were used as the sources of monoenergetic neutrons for this experiment: ${ }^{7} \mathrm{Li}(p, n){ }^{7} \mathrm{Be}$ for $0.57-$ and $1.0-\mathrm{MeV}$ ${ }_{\mathrm{Li}}(p, n)$ neutrons and ${ }^{3} \mathrm{H}(\mathrm{p}, \mathrm{n})^{3} \mathrm{He}$ for $1.5 \mathrm{MeV}$ neutrons. The pulsed proton beam (1-ns time width at a 2-ilHz repetition rate) was produced by the LoS Alamos Scientific Labcratory (LASL) vertical Van de Graaff generator. For 2-MeV protons, the lithium target was a $25-\mathrm{keV}$-thick deposit of ${ }^{7} \mathrm{LiF}$ on a gold disk. The tritium target was a $20-\mathrm{mm}$-long gas cell with a $5.3-\mathrm{mg} / \mathrm{cm}^{2}$ molybdenum entrance window and a gold beam stop. The energy spread of the neutrons from the gas cell was about $50 \mathrm{keV}$. 


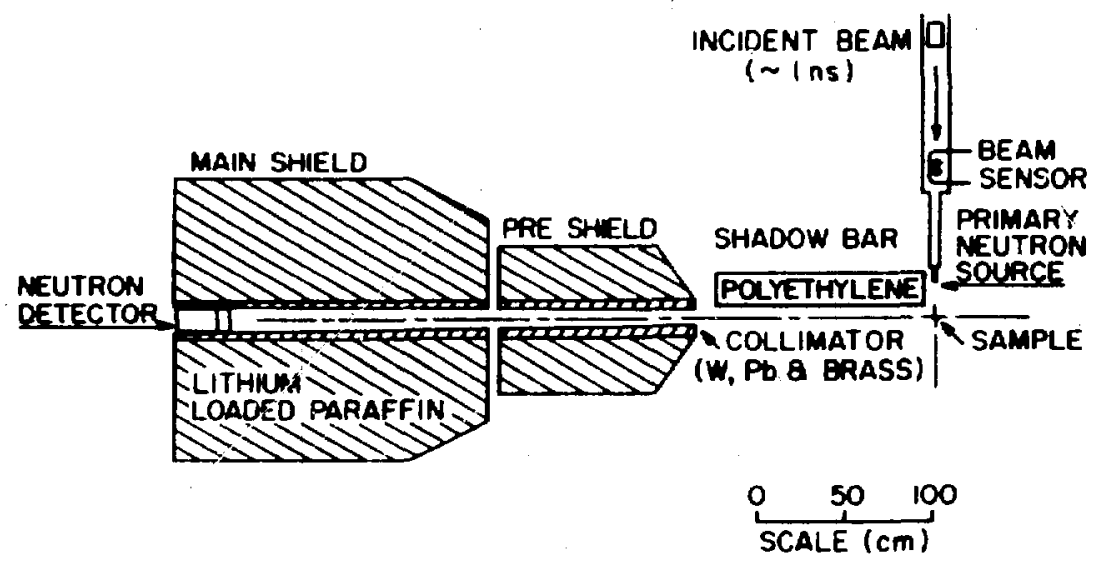

Fig. 1. Experimental arrangement for fast neutron TOF heasureli:ents.

E. ${ }^{242}$ pu sample

The plutonium sample, located $100 \mathrm{~mm}$ in front of the neutron target, consisted of six 6.4-rm-diam and 15-mm-high cylinders, with a total mass of $47.25 \mathrm{~g}$. These cylinders were placed in contact with the inner surface of a thin cylindrical 19-1:li-i.c. aluminum can. This arrangement was chosen so that the sample' would resenble a hollow right circular cylinder. During the experiment, the sample was rotated -1 rpm around its vertical axis to eliminate any angular dependence of the scattering caused by this unusual sample configuration. An empty aluminum can with the same dimensions as the one that held the plutonium was used to correct for neutrons scattered from aluminum.

\section{Neutron Detector}

Neutrons that emerged from the ${ }^{242} \mathrm{Pu}$ sample were detected with an NE 213 liquid scintillator positioned in a massive shield of tungsten and lithiumloaded paraffin. A standard pulse-shape discrimination circuit was used to reject gamma-ray pulses. The liquid scintillator was coupled directly onto the face of an RCA 8854 photomultiplier tube. A large polyethylene shadow bar shielded the detector from the target's direct neutron flux. The distance between the sample and the center of the scintillator was $2.28 \mathrm{~m}$. The reaction angle was changed by rotating the detector and shield around the center of the sample.

Two detector biases were used throughout the experiment. The higher bias was set at the pulse height minimum between the 26 - and $60-\mathrm{keV}{ }^{241}$ Am gammia rays as shown in Fig. 2. The TOF spectra of pulses between the higher bias and the 


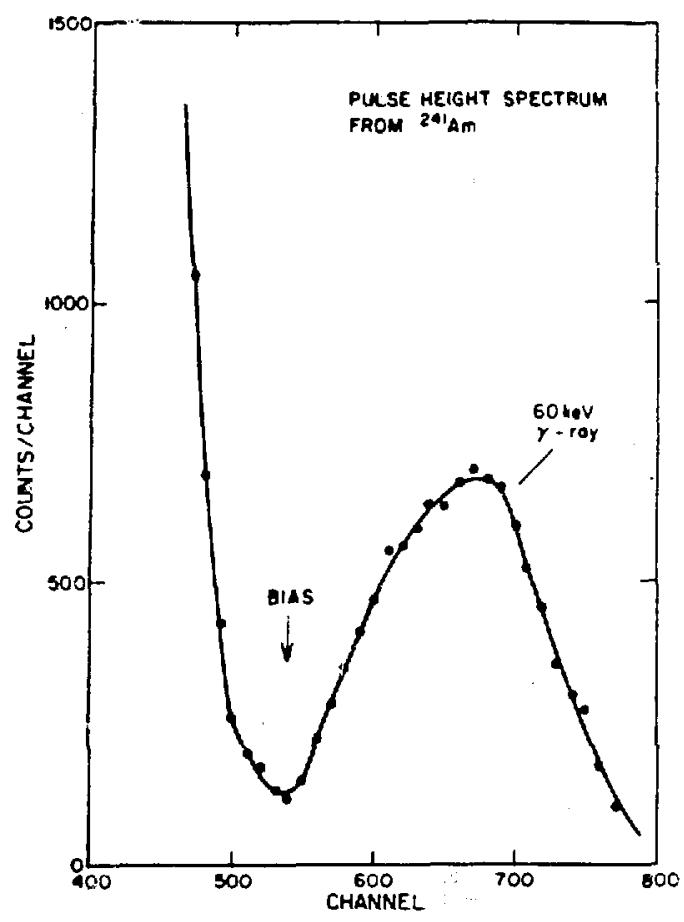

Fig. 2. The pulse height spectrum of 241 Am gamma rays in the NE 213 detector, showing the bias position.

lower bias were not useful because few neutrons of interest give rise to pulses in this energy range. We therefore analyzed only data for the higher bias. A small liquid scintillator detector, located $5 \mathrm{~m}$ from the target at $0^{\circ}$, monitored neutrons by TOF and was used, with a measurement of the target current, to normalize the measurements.

\section{DATA COLLECTION AND ANALYSIS}

The TOF spectra were obtained from an EG\&G TDC-100 time digitizer whose start and stop inputs were derived from the detector anode pulse and a beam sensor located just in front of the target.

The TOF spectrum of the empty aluminum can was used to correct for scattering from the aluminum can that held the plutonium. Before subtraction, however, the spectrum of the empty can had to be modified to account for neutran attenuation in the plutonium. After subtraction, the net spectrum always contained an excess number of counts because of the natural radioactivity of the plutonium sample. This constant background, which was determined from a region of the spectrum where only time-independent counts should occur, was subtracted from the raw spectrum. These corrections have already been applied to the $55^{\circ}$ TOF spectrum shown in Fig. 3 for incident neutrons of $0.57 \mathrm{MeV}$. 


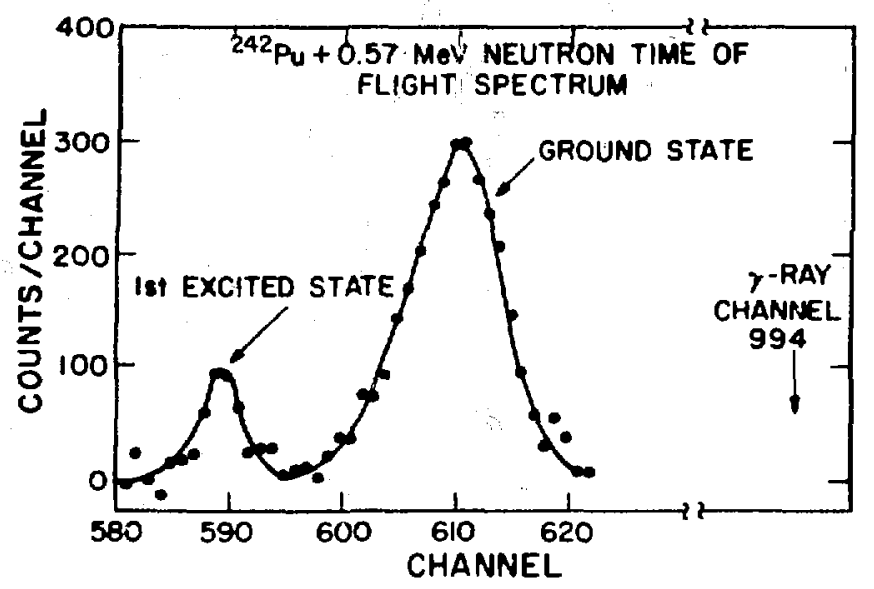

Fig. 3. TOF spectrum for $0.57-\mathrm{MeV}$ incident neutrons. Backgrounds from the empty aluminum can and the natural radioactivity of the sample have been subtracted.

Differential cross sections for the separable peaks can be computed in two ways. The first method uses a zero-degree measurement of the neutron flux and the equation

$\frac{d_{\sigma}}{d_{\Omega}}$

in which

$\frac{\mathrm{d} \sigma}{\mathrm{d} \Omega}$

Pu Counts

Pu Nuclei

$\mathrm{R}$$$
\text { (Counts at } \left.0^{\circ}\right)=\text { the number of }
$$

(Counts at $\left.0^{\circ}\right)=$ the number of

$\frac{d}{d_{0}}$

$\frac{\varepsilon\left(E_{0}\right)}{E\left(E_{\eta}\right)}$

M the samiple, $E_{j}$, and

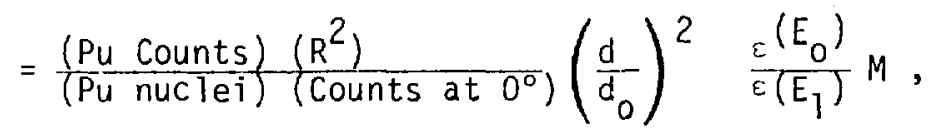

$=$ the differential cross section for the peak of interest,

$=$ the number of counts in the peak of interest from Pu,

$=$ the number of ${ }^{242} \mathrm{Pu}$ nuclei in the sample,

$=$ the harmonic mean of the distance from the target to

$=$ the ratio of the distances sample to detector and target to detector,

= the ratio of the neutron detection efficiences for source neutrons of energy $E_{0}$ and scattered neutrons of energy

$=$ the multiple scattering correction. 
The second method relates the plutonium differential cross section to the well-known cross section of neutron scattering by hydrogen through the equation

$$
\begin{aligned}
& \text { des } \\
& \overline{d \Omega} \\
& \text { in which } \\
& \mathrm{H} \text { Counts. = the number of counts in the peak of neutrons scattered by } \\
& \frac{d}{d} \frac{d}{\Omega}(n p) \quad=\text { the differential cross section for np scattering, and } \\
& \frac{\varepsilon\left(E_{G \mid}\right)}{\varepsilon\left(E_{P u}\right)} \quad=\text { the ratio of neutron detection efficiencies for energies } \\
& \text { neutrons scattered from plutonium. }
\end{aligned}
$$

We used both methods to compute cross sections for incident neutron energies of 0.57 and $1.0 \mathrm{MeV}$, and the results agree to within $4 \%$ for $0.57 \mathrm{MeV}$ and $2 \%$ for 1.0 MeV. For incident neutrons of $1.5 \mathrm{MeV}$, only Eq. (2) was used.

The relative efficiency of this detector is well known. 1 However, because the energies of the scattered neutrons are only slightly less than the energy of neutrons in the incident beam, only a small segment of the efficiency curve is needed.

Attenuation and multiple scattering corrections for elastically scattered neutrons were obtained fron neutron transport calculations made with the LASL Monte Carlo code MCNP. ${ }^{2}$ Attenuation accounts for a relatively constant $30 \%$ correction, whereas the multiple scattering correction is ang?e dependent at $\sim 2 \%$ at $20^{\circ}$ and $33 \%$ in the cross section minimum near $70^{\circ}$ for $1.5-$ MeV incident neutrons. Because the inelastic cross sections are almost isotropic, we used a procedure developed by Cranberg and Levin ${ }^{3}$ for heavy nuclei to calculate multiple scattering and attenuation corrections for differential inelastic scattering. This correction can be applied only to the angle-integrated cross sections for elastic scattering because the multiple scattering is angle dependent. We made these calculations also, and the corrections for the angleintegrated elastic scattering cross section agreed to within $4 \%$ of the Monte Carlo correction. 


\section{RESULTS AND DISCUSSION.}

Results of these measurements are shown in Figs. 4 through 6 and are 1 is ted in Table I. The errors listed in Table I are statistical in origin. Additional systematic errors were estimated to be 11,13 , and $12 \%$ for the $0.57-, 1.0-$, and 1.5-MeV data, respectively, and arise from uncertainties in normalization, efficiency, multiple scattering, and attenuation estimates. Because some of these errors vary with different experimental conditions, they are only approximate. Because few experimental results exist for ${ }^{242} \mathrm{Pu}$, comparisons are made with nearby elements having similar properties and an evaluation by Madland and Young ${ }^{4}$.

\section{A. Incident Neutrons of $0.57 \mathrm{MeV}$}

Angle integration of the elastic and inelastic ( $E^{*}=45 \mathrm{keV}$ ) cross sections (Fig. 4) results in cross sections of 5.9 and $1.3 \mathrm{~b}$, respectively. The elastic cross section agrees with that measured by Cranberg and Levint ${ }^{5}$ for ${ }^{238} U$ at 0.55 llev but is nearly 0.4 b higher than the measurement by Smith et al. ${ }^{6}$ for $240 \mathrm{Pu}$. The inelastic cross section is about $10 \%$ lower than the measured value for ${ }^{238} \mathrm{U}$ in the $45-\mathrm{keV}$ state. Summation of partial cross sections, $\sigma_{e l}=5.9 \mathrm{~b}$, $\sigma_{i n}=1.3+0.30 \mathrm{~b}$ (Ref. 5), $\sigma_{f}=0.4 b$ (Ref. 7 ), and $\sigma_{n, \gamma}=0.1 \mathrm{~b}$ results in $\mathrm{a}$ total cross section of $8.0 \mathrm{~b}$. This is also $\sim 0.5 \mathrm{~b}$ higher than the measured value for ${ }^{240} \mathrm{Pu}$ but agrees with the values in Brookhaven National Laboratory (BNL) report for ${ }^{238} \mathrm{U}$. The evaluated total cross section (Ref. 4) is higher than our measurement due primarily to 0.5 b more elastic scattering.

\section{B. Incident Neutrons of $1 \mathrm{MeV}$}

Angle integration of the elastic and inelastic ( $E^{*}=0.045,0.148 \mathrm{MeV}$ ) differential cross sections shown in Fig. 5 results in cross sections of 4.3 b for elastic scattering, $1.10 \mathrm{~b}$ for inelastic scattering from the 0.045 -MeV state and $0.42 \pm 0.10 \mathrm{~b}$ for scattering from the $0.148-\mathrm{MeV}$ state. Summation of these partial cross sections with $1.4 \mathrm{~b}$ for $(n, f)$ gives $7.3 \mathrm{~b}$ for the total section, which agrees with values of $7.5 \mathrm{~b}$ (Ref. 6) for ${ }^{240} \mathrm{Pu}, 6.7 \pm 0.6 \mathrm{~b}$ of Cranberg and Levin ${ }^{3}, 7.2$ b (Rei. 7) for $233_{u}$, and 7.2 b from the evaluation (Ref. 4). The total elastic cross section also agrees with these references. One would expect that the inelastic cross sections and the compound elastic portion of the elastic would be smaller than those for ${ }^{238}$ u because the fission channel is completely open for ${ }^{242} \mathrm{Pu}$. The $1-\mathrm{MeV} 90^{\circ}$ cross sections reported by Beghian et a1. ${ }^{8}$ for ${ }^{238} \mathrm{U}$ agree with the present data for the inelastic scattering but not for the elastically scattered neutrons. 


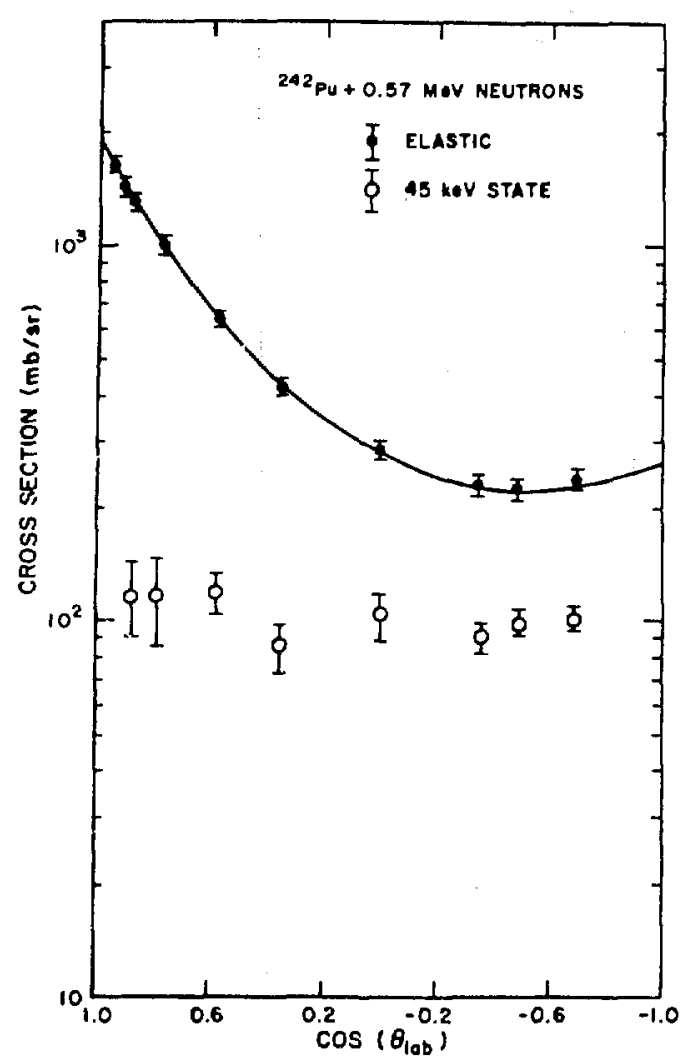

Fig. 4 .

Differential cross sections for the ground and first excited states of $242 \mathrm{Pu}+n$ for $0.57-\mathrm{MeV}$ incident neutron energy.

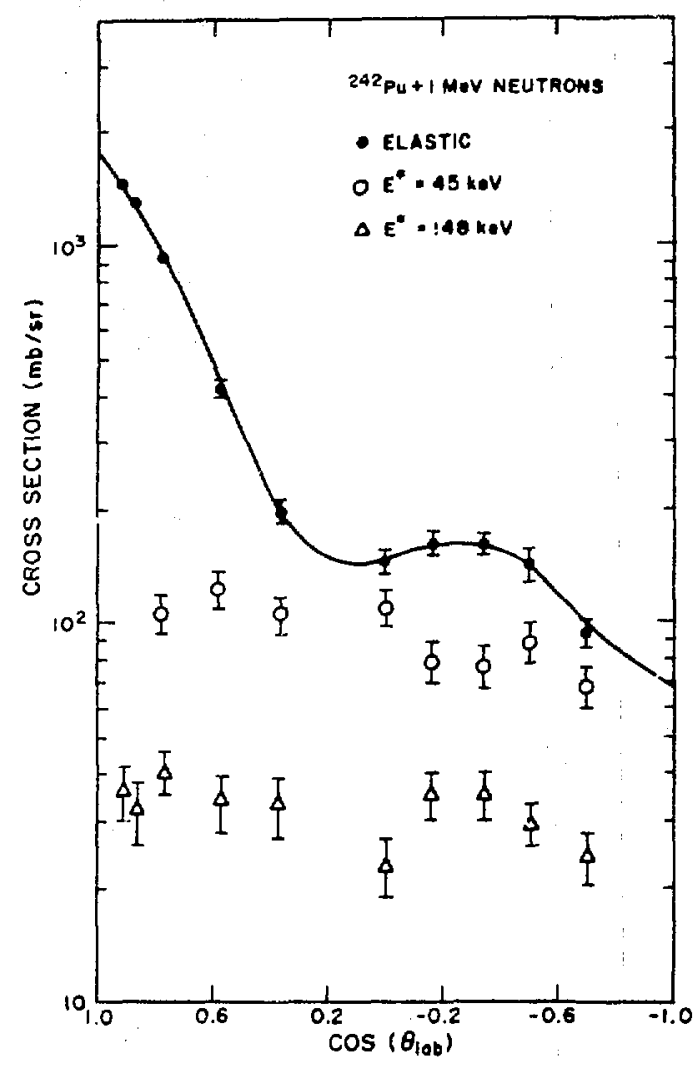

Fig. 5.

Differential cross sections for the ground, first, and second excited states of ${ }^{242} \mathrm{Pu}+\mathrm{n}$ for $1.0-\mathrm{MeV}$ incident energy.

\section{Incident Neutrons of $1.5 \mathrm{MeV}$}

It was impossible for us to separate elastically scattered neutrons from those inelastically scattered by low-lying excited states, so we analyzed the observed scattering as if it were one state. From these differential cross sections, we subtracted the $46 \mathrm{mb} / \mathrm{sr}$ that were estimated as the isotropic differential cross sections of the first two excited states.

As shown in Fig. 7, two other peaks are observed in the TOF spectra that correspond to excited states near $0.8 \mathrm{MeV}$ and $1.0 \mathrm{MeV}$, where another rotational band based on a $1^{-}$state begins and a second $0^{+}$state occurs. Each of these peaks has a cross section of $0.31 \pm 0.10 \mathrm{~b}$. Summation of the partial cross sections, $\sigma_{e}=3.94 \mathrm{~b}, \sigma_{i}=0.6+0.6=1.2 \mathrm{~b}$, and $\sigma_{f}=1.4 \mathrm{~b}$, gives $6.6 \mathrm{~b}$ for a total cross section. This also agrees with total cross sections of $7.0 \mathrm{~b}$ for ${ }^{238} \mathrm{Pl}_{\mathrm{l}}$ (Ref. 7), ${ }^{240} \mathrm{Pu}$ (Ref. 6), and ${ }^{242} \mathrm{Pu}$ (Ref. 4), al though we may have missed some weaker inelastic states in our summation. 

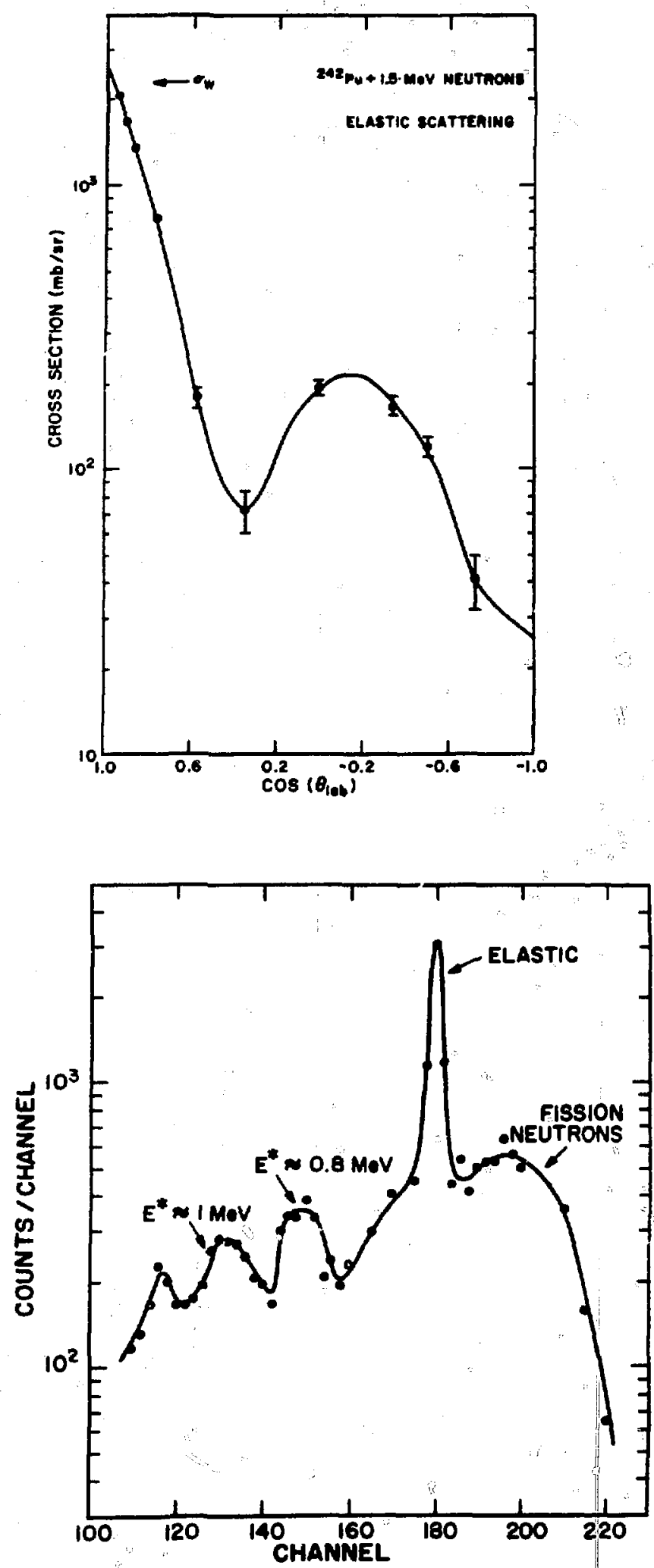

Fig. 6. Differential cross sections for the ground state of ${ }^{242} \mathrm{Pu}+\mathrm{n}$ at $1.5 \mathrm{MeV}$-incident energy.
Fig. 7. Neutron TOF spectrum for 1.5-MeV incident neutrons interacting with $242 \mathrm{Pu}$. The elastic peak includes the first and second excited states. This spectrum al so shows two peaks at $0.8-$ and 1.0-MeV excitation energy. 
TABLE I

LABORATORY CROSS SECIIIONS FOR NEUTRON SCATTERING FROM ${ }^{242} \mathrm{Pu}$

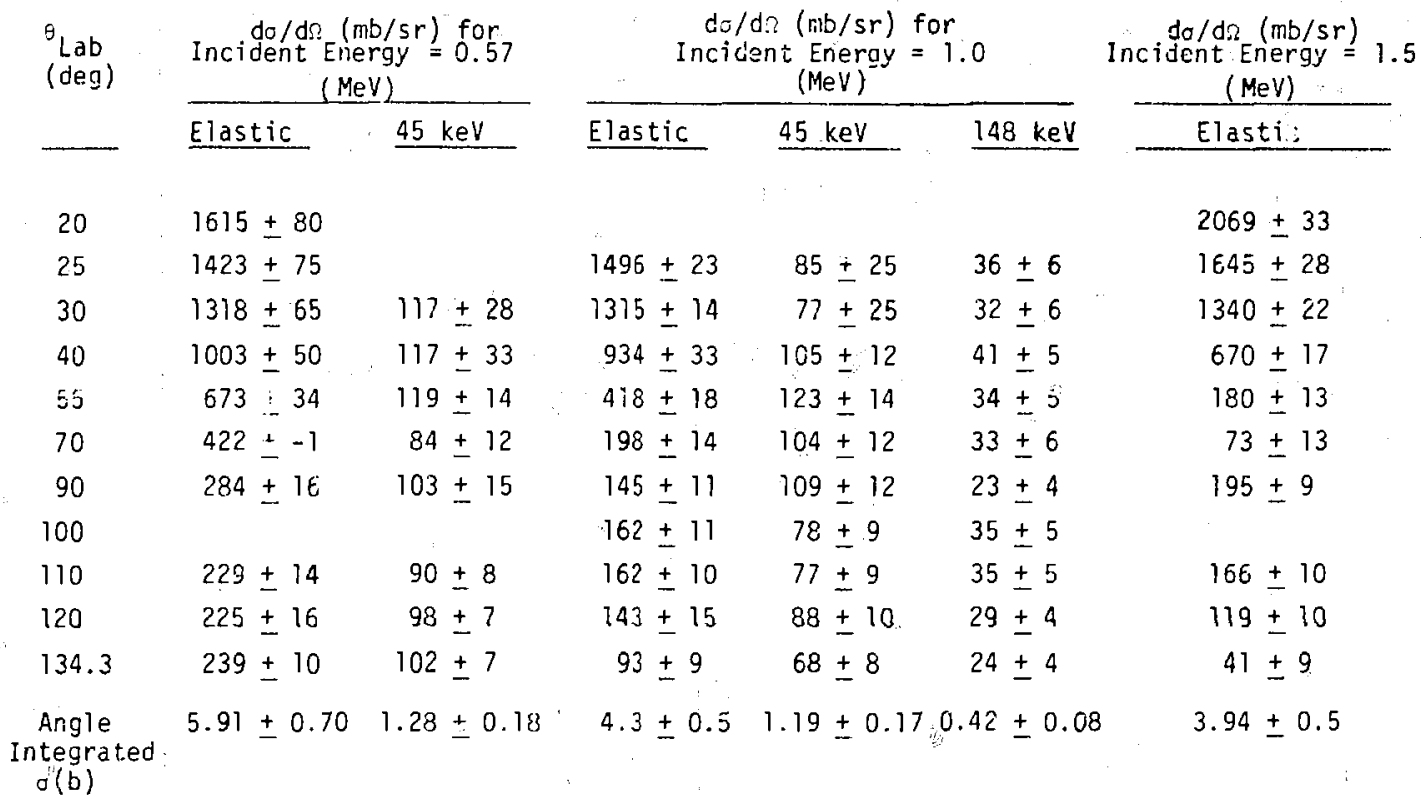

\section{REFERENCES}

1. M. Drosg, D. M. Drake, and P. Lisowski, to be published in Nucl. Inst. \& Meth.

2. E. O. Cashwe 1], I. R. Neergaard, W. M. Taylor, and G. D. Turner, "MCN: A Neutron Monte Carlo Code," Los Alamos Scientific Laboratory report LA-4751 (January 1972).

3. L. Cranberg and J. S. Levin, "Neutron Scattering by ${ }^{235} \mathrm{U},{ }^{239} \mathrm{Pu}$, and ${ }^{238} \mathrm{U}$," Los Alamos Scientific Laboratory report LA-2177 (January 1959).

4. D. G. Madland and P. G. Young, "Evaluation of $n+{ }^{242} P u$ Reactions from $10 \mathrm{KeV}$ to $20 \mathrm{MeV}, "$ Los Alamos Scientific report LA-7533-MS (October 1978).

5. L. Cranberg and J. S. Levin, "Inelastic Neutron Scattering by ${ }^{238} \mathrm{U}, "$ Phys. Rev. 109, 2063-2068 (1958).

6. A. B. Smith, P. Lambropoulos, and J. F. Whaten, "Fast Neutron Total and Scattering Cross Sections of $240 \mathrm{Pu}$," Nucl. Sci. Eng. 47, 19-28 (1972).

7. D. I. Garber and R. R. Kinsey, "Neutron Cross Sections, Volume II, Curves," Brookhaven National Laboratory report 325, 3rd Ed., Vol. II (1976).

8. L. E. Beghian, G. H. R. Kege1, T. V. Marcella, B. K. Barns, G. P. Couche11, J. J. Egan, A. Mittier, D. J. Pullen, and W. A. Schier, "Neutron Scattering Cross Sections of Uranium-238," Nuc7. Sci. Eng. 69, 191-110 (1979). 